\title{
A New MAC Protocol Design for Long-term Applications in Wireless Sensor Networks
}

\author{
Yunhuai LIU and Lionel M. NI \\ Department of Computer Science and Engineering \\ Hong Kong University of Science and Technology \\ \{yunhuai,ni\}@cse.ust.hk
}

\begin{abstract}
This paper presents the design, implementation and performance evaluation of a new MAC protocol, called A$M A C$, for wireless sensor networks. A-MAC combines the strengths of TDMA and CSMA to achieve the goal of low power transmissions for long-term surveillance and monitoring applications, where sensor nodes are typically vigilant for a long time and inactive most of the time until some event is detected. A-MAC employs an advertisement mechanism to eliminate collisions and reduce the overhearing and idle listening, which are the major energy wastes in wireless sensor networks. The distinctive feature of A-MAC is that a node needs to be active only when necessary as it is the transmitter or the receiver. During other times it can safely turn off its radio. Furthermore, to meet different application requirements, A-MAC supports two operation modes by which nodes can adaptively switch their operation modes according to the instant requirements and conditions of the network. A-MAC is implemented in TinyOS. By comparing A-MAC with existing MAC protocols, we show that A-MAC presents significant improvements in terms of power consumption and throughput.
\end{abstract}

\section{Introduction}

Wireless sensor network (WSN) is a fast emerging technology due to its wide range of potential applications [1]. Among all the design considerations, the power issue is one of the major concerns with a large community of researchers focusing on energy efficiency and conservation schemes.

Among all the components of a sensor node, the transceiver accounts for the majority of energy consumptions[2]. The power consumptions of some current sensor platforms, including Mica2, MicaZ and the newest Telos, are compared in 1 in the context of various operations.
Table 1. Powers of sensor platforms

\begin{tabular}{|l|r|r|r|}
\hline Operation & Telos & Mica2 & MicaZ \\
\hline MCU Idle & $54.5 \mu \mathrm{A}$ & $3.2 \mathrm{~mA}$ & $3.2 \mathrm{~mA}$ \\
MCU Active & $18 \mathrm{~mA}$ & $8.0 \mathrm{~mA}$ & $8.0 \mathrm{~mA}$ \\
Radio RX. & $21.8 \mathrm{~mA}$ & $15.1 \mathrm{~mA}$ & $23.3 \mathrm{~mA}$ \\
Radio Tx. (0dbm) & $19.5 \mathrm{~mA}$ & $25.4 \mathrm{~mA}$ & $21.0 \mathrm{~mA}$ \\
\hline
\end{tabular}

From this table, we find that Telos, the newest generation of sensor node, dramatically decreases the electronic currents in many fundamental states. The MCU Idle current is decreased from $3.2 \mathrm{~mA}$ to $54.5 \mu \mathrm{A}$. On the other hand, the power consumption of transmission and reception does not show such an obvious reduction. For Radio RX (reception), there is even a slight increase, from 15.1mA of Mica2 to $21.8 \mathrm{~mA}$ of Telos. This implies that with the advances of electronics, the power consumption of processing operations in sensor nodes can be dramatically decreased, while reducing the energy utilization for wireless communications is much harder. Therefore, we believe that the transceiver component will account for the major source of the energy consumption for a long time until significant changes in hardware design are made.

In wireless communications, there are four major sources of energy waste that significantly shorten the life time of a WSN [3-5]. The first one is collisions, where two nodes transmit at the same time and interfere with each other. The second source is overhearing, meaning that a node picks up packets destined to other nodes. The third source is idle listening. Note that in most of the current designs, sensors have to turn their radios on during the entire active time, waiting for possible incoming packets. The last source of energy waste is control packet overhead for protocols to exchange required information.

A fundamental observation is that the four energy waste sources play different roles in different application scenarios. For high data rate applications [8], the wireless channel may be saturated and congested, making collisions and 
overhearing the dominating factors of energy depletion. On the other hand, for low data rate applications (e.g., [9-11]), idle listening is often the case and may account for the major part of energy depletion. Ideally, a single approach is needed to target at all four energy consumptions in order to minimize the energy consumption in all kinds of application scenarios. This requirement is the main motivation for our work and such an approach is the primary design goal of this paper.

Energy conservation and efficient protocols can be addressed in different layers of the network. Recall that the Medium Access Control (MAC) layer has direct access to the radio control, making it one of the most appropriate positions to handle the radio-related problems [12]. Current wireless MAC protocols can be roughly classified as contention-based and schedule-based.

As a contention-based MAC protocol, CSMA [13-16] (e.g., 802.11x) is a popular MAC technique in wireless networks due to its simplicity, flexibility, and robustness. These advantages, however, come at the cost of collisions and high power consumptions. When the traffic load is high, the probability of collisions is high; therefore nodes may waste a large amount of energy on retransmission, back-off procedures, and overhearing. On the other hand, for low data rate applications where the traffic load is light, nodes spend most of their time and energy on idle listening.

In contrast to CSMA, scheduled-based TDMA (timedivision multiple access) [17-21] techniques offer an inherent collision-free scheme. The first advantage of TDMA is that interference between adjacent wireless links is guaranteed to be avoided. Thus, the energy waste coming from packet collisions is diminished. Second, TDMA can solve the hidden terminal problem without extra message overhead because neighboring nodes transmit at different time slots.

However, current TDMA techniques applied in sensor networks still have many limitations. First, current TDMA schemes fail to reduce the energy consumption of overhearing packets. As a result, a large amount of energy is wasted on useless overhearing. Second, TDMA often requires a centralized node to maintain the collision-free schedule which may not be practical in sensor networks.

In this paper, we propose a novel MAC protocol, namely A-MAC (Advertisement-based MAC), for sensor networks, aiming at providing collision-free, non-overhearing and less idle-listening transmission services. A-MAC is designed mainly for long-term surveillance and monitoring applications. In such applications, nodes are typically vigilant for a long time and inactive most of the time until something is detected. Some additional latency may be introduced at an acceptable range, while the life time of the WSN is dramatically prolonged.

A-MAC is a hybrid of CSMA and TDMA protocols. The main feature of A-MAC is that nodes are notified in advance when they will be the receivers of packets. A node is active only when it is the sender or the receiver, during other time it just goes to sleep. Through this means, energy wastage is avoided on overhearing and idle listening.

The main contributions of this paper are as follows: (1) we discuss MAC layer power consumption and energy waste issues in the context of sensor networks and drawbacks of current MAC protocols are identified; (??) we propose a novel MAC protocol, called A-MAC, for targeting these issues and give a thorough analysis of A-MAC. We implemented A-MAC on top of Berkeley Mica2 Motes [23]. We compare A-MAC with some existing MAC protocols thoroughly. Some early experiences are reported along with lessons that we have learned.

The remainder of this paper is organized as follows. In Section 2, we describe some existing power saving solutions in WSN. Then in Section 3, we present detailed design of A-MAC. Section 4 analyzes the impact of control parameters on the performance of A-MAC. We report the measurement results of A-MAC in Section 5. In the last section, we conclude our work and sketch some future research directions.

\section{Related Work}

MAC protocols for WSNs were rooted from conventional wireless protocols, especially the 802.11 families [13]. In 802.11-based protocols, wireless access is specified in both ad hoc environments known as Distributed Coordination Function and infrastructure systems called Point Coordination Functions. However, 802.11-based protocols are not suitable for WSNs. Not only are the networking conditions different, but the applications for WSNs have different demands.

S-MAC [4] is one of the first protocols specially designed for WSNs. Inspired by PAMAS [14], S-MAC attempted to address the energy issues by periodical operations of the transceiver. The core idea of S-MAC is to divide the time dimension into active periods and sleep periods. Nodes can fully function in the active periods, while turning off their radios in the sleep periods. The main drawback of S-MAC is its inability to adapt to the network environment. For scenarios of dense deployment or high traffic loads, more collisions may be introduced and the throughput is significantly decreased. On the contrary, while the traffic load is light, a large portion of energy is spent on idle listening. However, S-MAC presents us a good starting point for our research. T-MAC [5] improves the energy usage of S-MAC by introducing more sleep time in the transmission period. It applies a short listening window at the beginning of each active period.

B-MAC [26] has another design philosophy that al- 


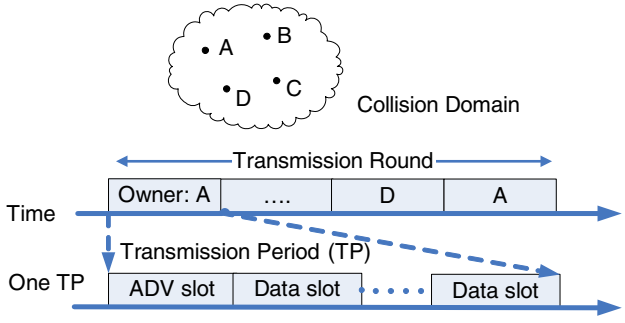

Figure 1. Structure of A-MAC

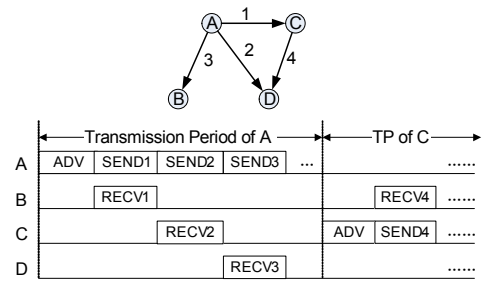

Figure 2. An example of transmission periods

lows applications to implement their own MAC protocols through a well-defined interface. It adopts LPL (low power listening) and a clear channel assessment technique to improve the channel utilization. B-MAC is shown to have higher throughput and better energy efficiency than that of S-MAC and T-MAC. The core philosophy of B-MAC is to transform the energy waste at the receiver side to the sender side. Each time before transmission, the sender has to use a long preamble to wake up the receiver; the larger the duty cycle of the receiver the longer the permeable should be used.

Z-MAC [12] is a hybrid MAC protocol using CSMA as the baseline with a TDMA scheduler to enhance the contention resolution. In Z-MAC, a time slot assignment protocol DRAND [28] is performed in the initialization phase. DRAND guarantees that each node is assigned with a unique time slot within its two-hop neighbors. In our paper, we also apply the DRAND protocol to assign nodes unique time slots to avoid collisions. Interested readers may refer to [28] for detailed information about the DRAND protocol. With the assistance of DRAND, Z-MAC increases the throughput to some extent and can be applied in realtime applications. However, Z-MAC lacks a mechanism by which nodes can be notified in advance in which time slot they will be the receiver. Accordingly, nodes have to keep sensing and listening in all time slots, thus wasting a large amount of energy on overhearing and idle listening. To reduce overhearing and idle listening is the main objective of our paper - nodes are active only when necessary; otherwise, they are sleep. There is guaranteed no collision, no overhearing, and less idle listening.

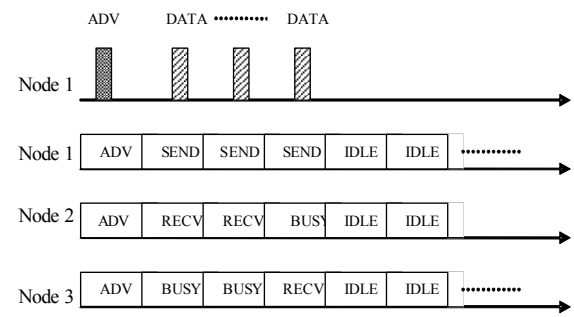

Figure 3. An example of Transmission Schedule

Table 2. Notations used in this paper

\begin{tabular}{|l|l|}
\hline Notation & Description \\
\hline$T_{P}$ & The length of the transmission period \\
\hline$T_{S}$ & The length of the ADV time slot \\
\hline$T_{D}$ & The length of the data time slot \\
\hline$N$ & The number of data time slots in one $T_{P}$ \\
\hline$R_{1}$ & Maximum transmission rate of A-MAC \\
\hline$R_{a p p}$ & Application sample rate. \\
\hline$L_{s y n c}$ & The length of the transmission round. \\
\hline$D$ & Average transmission delay of single packet \\
\hline$T_{c s}$ & Average carrier sense delay \\
\hline$T_{q}$ & Average queuing delay \\
\hline$T_{t x}$ & Average transmission delay \\
\hline
\end{tabular}

\section{A-MAC Description}

In this section, we present our A-MAC design. First, we give some definitions and notations used in later subsections. Some of the major design considerations and principles are also discussed. In the last three subsections, we present the A-MAC design and its two operation modes in detail.

\subsection{Design principles}

\subsubsection{Concepts and notations}

The core idea of A-MAC is TDMA-based with CSMA as the baseline MAC scheme to enhance the accessibility of the wireless channel.

Basically, A-MAC allocates each node a certain number of unique time slots within its two-hop neighbors. Nodes then make use of these pre-assigned time slots to transmit the data packets without interference with others. A-MAC also has an advertisement scheme to allow the sender to notify its neighbors about its transmission schedule. With this scheme, only the receiver needs to be active during the transmission and other nodes can safely go to sleep. In AMAC, a node needs to be active only when it is the sender or the receiver. At other times it just goes to sleep. 


\subsubsection{Definitions and notations}

As illustrated in Fig. 1, the time dimension is divided into a number of fixed length transmission periods, where the length of each transmission period is denoted as $T_{P}$. Nodes are synchronized and each of them is assigned with a transmission period being unique within its two-hop neighborhood. Mostly, they can send out their packets only in their own transmission period. Thus, in theory, it guarantees collision free. The node which is assigned the transmission period is called the owner of the period. Note that mostly only the owner node can be the sender in each transmission period. The transmission period assignment algorithm and the transmission modes will be described later.

Traditional TDMA-based MAC designs follow a similar idea that the time dimension is divided into many small time slots. In our A-MAC design, however, the transmission period is relatively long so that nodes are allowed to transmit multiple packets in its own time period. More advantages of this longer period design will be shown later.

Definition ADV is an advertisement packet sent by the owner to notify its neighbors of its transmission schedule.

At the beginning of each transmission period, the owner sends an ADV packet carrying its own transmission schedule in this period. All other nodes shall be active to receive this ADV packet so that they will know who will be the receiver and when the receiver needs to be active.

Definition Advertisement time slot is a small time slot at the beginning of each transmission period. It allows the owner to send the ADV packet and non-owner to receive the ADV packet. The length of the advertisement time slot is denoted as $T_{S}$.

Definition Data time slot is a small portion of a transmission period after the advertisement time slot, allowing the owner to send data packets. The length of the data time slot is denoted as $T_{D}$.

As illustrated in Fig. 1, a transmission period is composed of one advertisement time slot and several data time slots. Let $N$ denote the number of data time slots within a transmission period. Then we have the relation that:

$$
T_{P}=T_{S}+N \times T_{D}
$$

Definition Transmission round is the cycle of a node to be the owner of the transmission period. The length of the transmission round is denoted as $L_{\text {sync }}$.

\subsection{Protocol overview}

In this subsection, we present the basic operations of AMAC. As exemplified in Figure 1, the time dimension is divided into many transmission periods owned by different nodes.

In each transmission period, a node first enters the advertisement time slot to send or receive ADV packets. The node then enters data time slots to send or receive data packets. After all data time slots, the owner will be silent. Now it is the new owner's turn to send the ADV packet and data packets, and the whole process is repeated. A node will become the owner again after some others' transmission periods.

Figure 1 illustrates an example of a collision domain with four nodes, namely node A, B, C and D. Node A is the owner of the first transmission period. It will become the owner again after three transmission periods. Hence, $L_{\text {sync }}$ the length of the transmission round is equal to 4 . $L_{\text {sync }}$ is a critical parameter that greatly impacts the performance of A-MAC, which is mainly determined by the network density. Detailed information about $L_{\text {sync }}$ is given later.

Figure 2 gives another example of transmissions in AMAC. Similarly, four nodes A, B, C and D are in the collision domain. Node A sends out three packets 1, 2 and 3 in its transmission period. Node B, C and D are the corresponding receivers. The bottom part of Fig. 2 depicts the state sequences of these nodes. Note that in the ADV slot all nodes are active; while in the other data time slots, only the receiver is active.

A-MAC is empowered by collision free, nonoverhearing and little idle listening features while sacrificing the control overhead on ADV packets. In AMAC, a node can emit up to $N$ data packets for each ADV control packet. The node then waits $L_{\text {sync }}-1$ transmission periods for another transmission round. Therefore, the key issue that has the most crucial impact on the performance of A-MAC is the parameter $N$ and $L_{\text {sync }}$. Note that $N$ is adjustable, while $L_{\text {sync }}$ is an environment dependent parameter determined mainly by the network density. We give further analysis on $N$ and $L_{\text {sync }}$ in later sections.

\subsection{Transmission modes}

WSNs are application dependent computing environments. Different applications may have distinctive requirements on the power consumption, lifetime concerns, and quality of services. In order to design a MAC protocol subject to all these constraints, we propose two operation modes of A-MAC instead of a single, generic mode. Nodes can be freely switched between these two modes on-line, according to the specific requirements of different applications.

Definition Power Saving Mode (PSM): The power saving mode is mostly designed for applications where the power issue is the most concern and a maximized lifetime is the primary goal such as habitat monitoring systems. 
Definition Adaptive Contention Mode (ACM): The adaptive contention mode is designed for delay sensitive applications. More energy is allowed to be consumed while the transmission delay is tightly bounded.

In the last, we should mention how to assignment tranasmission periods. A-MAC runs a transmission period assignment algorithm DRAND which is a fully distributed algorithm. It guarantees that no node will choose the same transmission period as its two-hop neighbors. Since this part is not our contribution, we do not give too much details.

\section{A-MAC Analysis}

In this section, we give some analysis results of A-MAC. We first determine the basic time division unit $T_{S}$ and $T_{D}$, then calculate the upper limit of the transmission rate. Next, we derive the average transmission delay and the energy efficiency with effects of different parameters. Table 2 lists some of the notations in this section.

\subsection{Determining the value of $T_{S}$ and $T_{D}$}

As the fundamental unit to divide the time, $T_{S}$ and $T_{D}$ are largely dependent on applications, more specifically, the network deployment and the application.

To explore an optimal value of $T_{S}$, our target is to seek an upper bound of the ADV packet size that suitable for most settings of WSNs. Due to the space limitation, we omit the intermediate results. Finally, we suggest $T_{S}$ to be $40 \mathrm{~ms}$ based on the transmission rate of MICA2. A little more time is reserved for the purpose of robustness.

\subsection{Upper limit of transmission rate $R$}

In this subsection, we would like to derive the upper limit of the transmission rate $R$ that A-MAC PSM can offer

To avoid traffic overflow, the upper layer should not impose data that exceeds this rate. Here, we assume that only one packet will be -transmitted in each time slot. In one transmission round, each node owns one transmission period to transmit its data. Up to $\mathrm{N}$ data packets can be sent out. The length of transmission periods is $T_{P}$, and there are $L_{\text {sync }}$ transmission periods in one transmission round. Obviously, the upper limit of transmission rate $R$ is: $R=$ $\frac{N}{T_{P} \cdot L_{s y n x}}$. From Eq. (1), we have $T_{P}=T_{S}+N \times T_{D}$. Replacing $T_{P}$, we have:

$$
R=\frac{N}{\left(T_{s}+N \times T_{D}\right) \times L_{\text {sync }}}
$$

Figure 4 shows the relation between $R$ and $N$ with respect of different $L_{\text {sync }}$.

\subsection{Average transmission delay}

This subsection analyses the latency of A-MAC PSM, and quantifies the introduced delay. For a packet moving through a wireless network, it experiences the following delays as specified in [6]: Carrier sense delay, Back off delay, Transmission delay, Propagation delay, Processing delay, and Queuing delay.

Due to space limitation, we omit the intermediate results. Given a transmission, the expected packet delay is: $E(D)=\frac{1}{2} \times\left(T_{S}+N \cdot T_{D}\right) \cdot\left(1+T_{D} \cdot R_{a p p}\right) L_{s y n c}+T_{S}-T_{D}$. And the average delay is: $\operatorname{mean}(D)=\operatorname{mean}\left(T_{c s}+T_{t x}+\right.$ $\left.T_{q}\right)=T_{c s}+T_{t x}+E(D)$ Figure 5 shows the relation between the parameter $N$ and mean(D) with respect of different $L_{\text {sync }}$.

\subsection{Energy efficiency of A-MAC}

Although decreasing the value $\mathrm{N}$ can reduce the transmission delay, more energy is spent on ADV slots.

Figure 6 depicts the relation between the value $N$ and the energy spent on ADV time slots. Different curves represent different settings of the network density and thus the $L_{\text {sync }}$ value. This figure shows that the dominating factor is the number of data time slots $N$ rather than the network density. From the above analysis, we find that there is a trade-off between the delay and the power consumption when choosing the parameter $N$. A small value of $N$ gains a shorter delay, while more energy is used for control packet exchanges and vice versa. In real settings, applications may have different requirements for the delay and energy consumption. Application designers should evaluate the trade-offs and select an appropriate $N$ to fit their needs.

\section{Performance Evaluation}

In this section, we report some experimental results. We first introduce the testbed setups and the evaluation metrics. We then present the measurement results according to these metrics. We implement A-MAC based on TinyOS [21]. Mica2 [20] wireless sensor nodes are used as the hardware platform for our tests. A-MAC is compared with S-MAC. In these tests, S-MAC uses the default duty cycle $10 \%$.

\subsection{Experimental Setup}

In our experiments we apply three testbed setups: onehop, two-hop (nodes-sink), two-hop (nodes-relay-sink).

To evaluate the performance of S-MAC as well as AMAC, the following evaluation metrics are applied:

Energy consumption: Energy consumption is measured in the whole transmission process, including sleeping, idle, receiving and transmitting. 


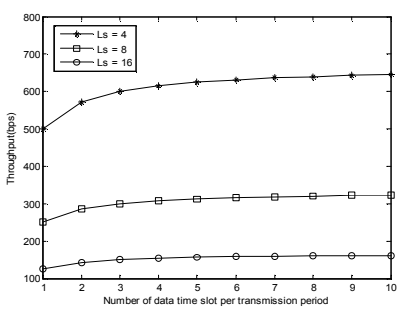

Figure 4: Relation between $N$ and $R$

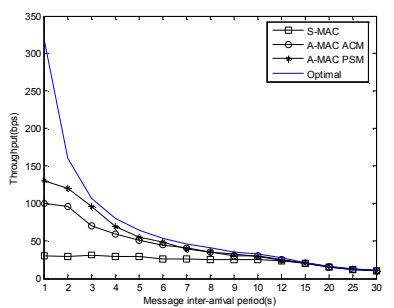

Figure 8: The throughput in one-hop topology

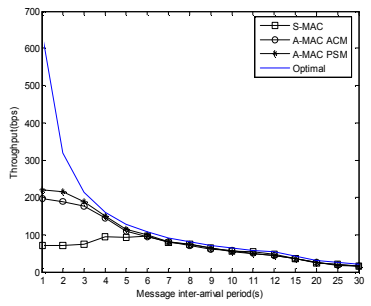

Figure 11: The throughput in the twohop topology

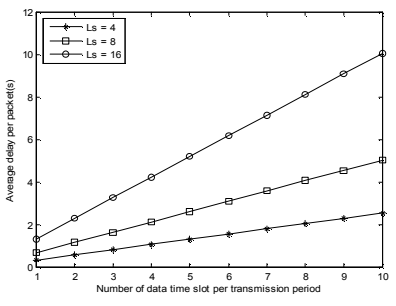

Figure 5: Relation between $N$ and the transmission latency

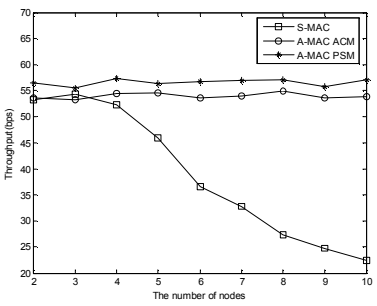

Figure 9: The throughput in different densities

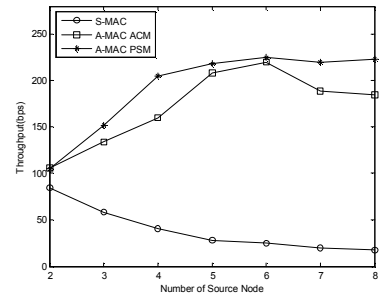

Figure 12: The throughput when the number of source nodes varies

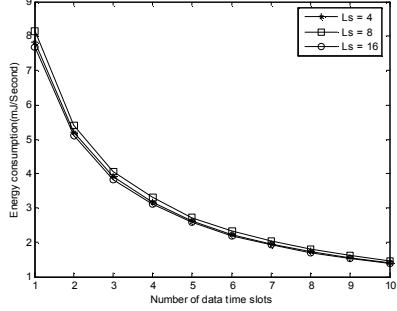

Figure 6: Relation between $N$ and the energy spent on ADV time slot

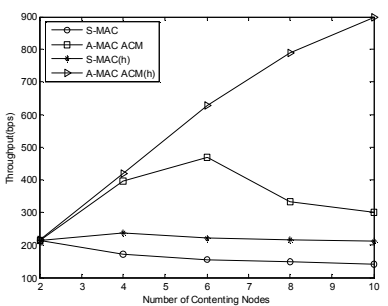

Figure 10: The throughput when hidden terminal exist

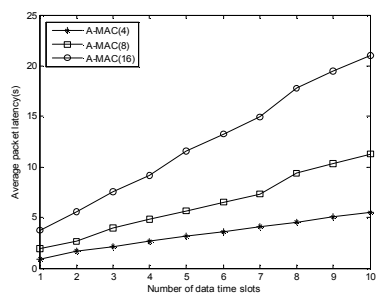

Figure 13: The delay as a liner function of the value $N$ and $L$
Delay: The delay is defined as the time difference between the packet generation and the arrival.

Throughput: To evaluate the throughput of different MAC protocols in different environments, we vary the contention level by changing the number of nodes and the transmission rate of each node.

Figure 8 and Figure 9 show the experimental results in a one-hop topology without hidden terminals. As we can see, A-MAC could maintain good throughput at different contention levels while the performance of S-MAC will degrade as the contention level increases. That is because, in A-MAC, each node does not need to compete with other nodes due to the collision-free schedule which guarantees that each node is assigned a dedicated segment of time to transmit. Therefore, increasing the number of nodes will not lead to a decrease in throughput. On the other hand, in S-MAC, nodes have to spend more time and also energy on back off procedures, which will ultimately lead to a decrease in throughput.

In the two-hop tesetbed, we measure the throughput when hidden terminals are present. The results of the nodessink topology are shown in Figure 10.

As we can see A-MAC ACM and S-MAC are sensitive to the hidden terminal problem while A-MAC PSM performs stable, maintaining a good throughput. However, since the transmission power is low, the achievable throughput also gets lower in A-MAC PSM.

Figure 11 shows the throughput of the two-hop network (5.2). The result is similar, meaning that A-MAC has a consistent behavior for two-hop transmissions.

Figure 12 shows the measurement throughput of S-MAC and A-MAC under different numbers of source nodes in the 
two-hop topology. In this testbed, we fix the message interarrival period of each node to be 6 seconds. From the results we can see that, in A-MAC, the actual throughput of the relay node will increase as the number of source nodes increases while the throughput of S-MAC drops as the number of source nodes increases since more collisions will be introduced.

\section{$5.2 \quad$ Latency}

The goal of this test is to reveal the impact of the value $N$ and $L_{\text {sync }}$ to the transmission delay, as we have analyzed earlier. We measure the average transmission delay by varying the value $N$ under certain $L_{\text {sync }}$. From Figure 13, we can see that as the value $N$ increases, a longer transmission delay will be introduced and a larger value of $L_{\text {sync }}$ will make this increase more aggressive. Our measurement results fit the previous analysis results (Figure 5).

\subsection{Energy consumption}

To evaluate the power consumption of A-MAC, we measure its energy utilization and compare with that of S-MAC in different scenarios. The measurement results are shown in this section.

Figure 14 shows the measurement results of energy consumption per second of each node in one-hop topology. The power consumption of S-MAC is almost independent of the traffic load due to its constant duty cycle. A-MAC PSM experiences better power saving performance than SMAC, especially when the traffic load is light. It is because in such case, idle listening becomes a major part of the power usage. In PSM, a node only needs to be active whenever it is the sender or the receiver. During other time it is asleep so that energy consumption for idle listening can be dramatically saved. From this figure, we also find that A-MAC ACM experiences the highest power consumption compared with the others. Recall that ACM allows nonowner nodes to compete for the idle data time slots, implying that they have to be in active in these slots and thus idle listening power cannot be saved.

Figure 15 shows the energy spent on each byte in different MAC protocols. Again we can find that A-MAC PSM successfully achieves the goal of energy efficiency due to its non-overhearing and less idle listening properties. Figure 15 also demonstrates that A-MAC ACM has a similar energy per byte rate to that of S-MAC. Recall that ACM has a much higher throughput comparing with S-MAC. Thus, even the power consumption is higher; they will still consume similar energy for each byte.

Figure 16 and Figure17 show the energy usage of the relay node in the two-hop topology under different traffic loads. The results are similar to the previous one in the one-hop topology, meaning that A-MAC PSM can achieve better performance than the other two.

Figure 18 and Figure 19 show the relation between the energy and the network density. We can find that as the number of nodes increases, both S-MAC and A-MAC PSM maintain a stable power consumption rate while that of AMAC ACM will increase. However, in S-MAC, since the throughput will drop as the density increases, the energy spent on each byte increases dramatically. The power consumption and the throughput of PSM will not be affected by the density. Thus the energy per byte rate will remain relative stable. ACM can maintain the same throughput even when the density increases, but more energy will be wasted on idle listening and overhearing. Therefore, both the energy per second rate and energy per byte rate will increase as the network density increases.

\section{Conclusions}

This paper presents A-MAC, a medium access control protocol specifically designed for WSNs. As energy efficiency is the primary goal in the protocol design, A-MAC obtains significant energy savings compared with other protocols by a collision-free, non-overhearing and less idle listening scheme. Consequently, the proposed A-MAC is able to greatly prolong the network lifetime, which is critical for practical sensor network applications. Two modes for A-MAC are proposed to meet various application requirements due to the nature trade-off between power consumption and transmission delay. This enables nodes to adaptively switch its operation mode according to the application.

We implemented a prototype system of A-MAC on top of Mica2 motes. Our experimental results show that AMAC can outperform existing WSN media access protocols in terms of throughput and power consumption and the experimental results have verified our design principle.

\section{References}

[1] D. Culler, D. Estrin, and M. Srivastava, "Sensor Network Applications," IEEE Computer, pp. 41-78, 2004.

[2] V. Raghunathan, C. Schurgers, S. Park, and M. B. Srivastava, "Energy-aware wireless microsensor networks," Signal Processing Magazine, vol. 19, pp. 40-50, 2002.

[3] M. L. Sichitiu, "Cross-Layer Scheduling for Power Efficiency in WSNs," In Proc. of INFOCOM,2004.

[4] J. H. Wei Ye, Deborah Estrin, "Media Access Control With Coordinated Adaptive Sleeping for WSNs," TRANSACTIONS ON NETWORKING, 2004.

[5] K. L. Tijs van Dam, "An Adaptive Energy-Efficient MAC Protocol for WSNs," In Proc. of SenSys'03,2003.

[6] S. Cui, A. J. Goldsmith, and A. Bahai, "Energyefficiency of MIMO and Cooperative MIMO Techniques in Sensor Networks," IEEE JSAC, vol. 22, 2004. 


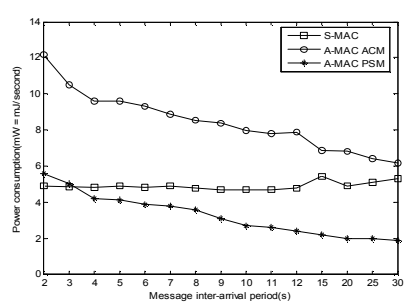

Figure 14: Power consumption in the one-hop topology

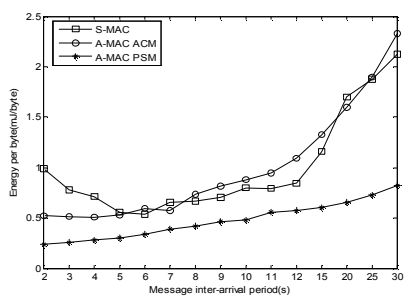

Figure 17: Energy efficiency of the relay node in the two-hop topology

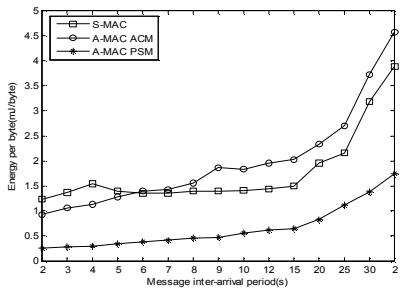

Figure 15: Energy efficiency in the one-hop topology

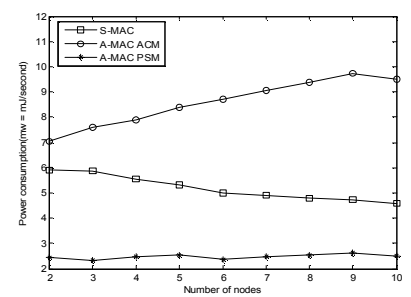

Figure 18: Power consumption as the number of nodes varies

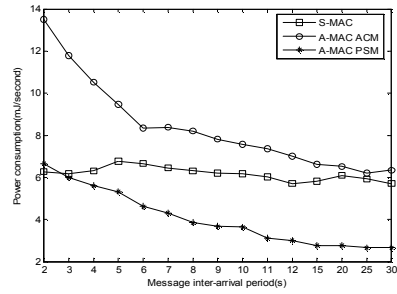

Figure 16: The power consumption of the relay node in the two-hop

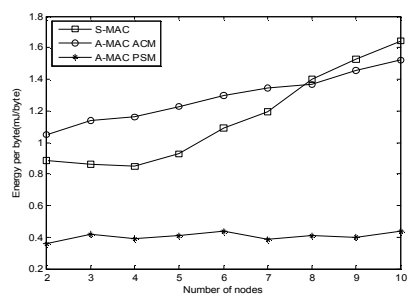

Figure 19: Energy efficiency as the number of nodes varies
[7] R. H. K. M. Stemm, "Measuring and reducing energy consumption of network interfaces in hand-held devices," IEICE Transaction, 1997.

[8] B. Hari, "Opportunities and Challenges in High-Rate WSNing," In Proc. of NSF NOSS Principal Investigator and Informational Meetings, October,2004.

[9] J. E. A. Cerpa, D. Estrin, L. Girod, M. Hamilton, J. Zhao, "Habitat monitoring: Application driver for wireless communications technology," In Proc. of ACM SIGCOMM workshop, 2001.

[10] M. A. M. Hamilton, D. Estrin, J. Rottenberry, P. Rundel, M. Srivastava, S. Soatto, "Extensible sensing system: An advanced network design for microclimate sensing," http://www.cens.ucla.edu.

[11] J. P. A. Mainwaring, R. Szewczyk, D. Culler, J. Anderson, "WSNs for habitat monitoring," In Proc. of 1st ACM International Workshop on WSNs and Applications,2002.

[12] A. W. Injong Rhee, Mahesh Aia, Jeongki Min, 'ZMAC:a Hybrid MAC for WSNs," In Proc. of SenSys'05,2005.

[13] "Wireless LAN Medium Access Control (MAC) and Physical Layer (PHY) Specification,"

[14] C. S. R. S.Singh, "PAMAS:power aware multi-access protocol with signalling for ad hoc networks," In Proc. of ACM Comput. Commun. Rev,1998.

[15] A. D. V.Bharghavan, S. Shenker, L. Zhang, "MACAW: A media access protocol for wireless lans," In Proc. of ACM SIGCOMM,1994.

[16] D. C. A. Woo, "A transmission control scheme for me- dia access in sensor networks," In Proc. of MobiCom,2001. [17] J. C. Haartsen, "The Bluetooth radio system,"

[18] A. Savvides, H. Park, and M. Srivastava, "The Bits and Flops of the N-hop Multilateration Primitive for Node Localization Problems," In Proc. of WSNA,2002.

[20] M. Y. K. Arisha, M. Younis, "Energy-aware TDMAbased MAC for sensor networks," In Proc. of IEEE Workshop on Integrated Management of Power Aware Communications, Computing and NeTworking (IMPACCT 2002),2002.

[21] T. Inukai, "An efficient SS/TDMA time slot assignment algorithm," IEEE Trans. Communications, 1979.

[22] G. L. J. Li, "A bit-map-assisted energy-efficient MAC scheme for WSNs," In Proc. of (IPSN04), April,2004.

[23] B. University of California, "Mica2 schematics" [24] B. University of California, "TinyOS CVS Repository at SourceForge," http://sf.net/projects/tinyos/,2004.

[25] I. F. Akyildiz, W. Su, Y. Sankarasubramaniam, and E. Cayirci, "A Survey on Sensor Networks," IEEE Communications Magazine, 2002.

[26] J. H. Joseph Polastre, David Culler, "Versatile Low Power Media Access for WSNs," In Proc. of SenSys, 2004. [28] A. W. Injong Rhee, Mahesh Aia, Jeongki Min, "DRAND: Distributed TDMA Scheduling for Sensor Networks," In Proc. of MobiHoc, 2006 